\title{
Effect of packer design on hydraulic fracturing of coal seam
}

\author{
Sergei Klishin ${ }^{1 *}$, and Vladimir Klishin ${ }^{2}$ \\ ${ }^{1}$ N.A. Chinakal Institute of Mining, Department of Deformable Solids and Granular Media \\ Mechanics, Novosibirsk, 630091, Russia \\ ${ }^{2}$ Institute of Coal, Federal Research Center for Coal and Coal Chemistry, Kemerovo, 650065, Russia
}

\begin{abstract}
The article addresses the issue of in-situ methane drainage from coal seams using multiple hydraulic fracturing. The authors undertake the stress-strain analysis of rock mass in the vicinity of a drainage borehole subjected to loading by sealing packers using the finite element method. The pressures at the packer and borehole wall contacts are calculated. It is shown how the length and spacing of the packers influence the values of the maximum axial tensile stresses on the borehole wall.
\end{abstract}

\section{Introduction}

One of the major trends in underground coal mining is engineering of safe and efficient facilities for coal extraction. Many mines are gas-hazardous, which impedes fast rates of face advance. Safe mining at increased face capacities requires reliable methods and special-purpose equipment for effective gas drainage of coal seams.

By analogy with oil reservoir engineering, coal mining practices use the method of direct treatment of coal seams by hydraulic dissection via gas drainage boreholes drilled from underground roadways [1-3].

The earlier research $[4,5]$ considered the process flow chart of multiple hydrodynamic effect exerted on coal seams. The two-sided packer design was also presented, and the labscale testing data on axial displacement of the packers were presented. Such treatment results in induced fracturing of coal seam, including opening of the existing cracks under the influence of fracturing fluid pressure. Naturally, the created fractures grow in the direction which is energy beneficial, and depends on stress state and natural jointing of rock mass. The existing cracks along the coal seam strike are closed under the action of gravity. Therefore, the main objective of hydraulic fracturing is creation of cracks oriented across the drainage boreholes.

The crosswise direction of cracks is the best for gas drainage. Multiple and high density created fractures in a single borehole expand the gas drainage area by hundreds of times. Under the action of the fracturing fluid pressure, the created fractures open wider and connect with natural cracks intact by the borehole. It is shown in [6] that initiation of an axial fracture or a cross-wise fracture is governed by the length $L$ of the isolated cavity between the packers and by the borehole diameter $d$ : when $L<2 d$, a single crosswise crack

\footnotetext{
*Corresponding author: sv.klishin@gmail.com
} 
is initiated, when $2 d<L<4 d$, many differently orientated fractures appear, and when $L>$ $4 d$, a single axial crack is created.

\section{Numerical stress-strain analysis of borehole wall loaded by packers}

\subsection{State of the art in hydraulic fracturing modeling}

The stress-strain behavior of rock mass, as well as the process of initiation and propagation of longitudinal or transversal created cracks in hydraulic fracturing using packers is the subject of numerous studies. These studies generally use the classical theory of elasticity, with numerical calculation of the response of geomedium to the preset boundary conditions $[7,8]$. In such problems, the boundary conditions are formulated by setting the normal and shear stresses exerted by fluid on geomedium $[9,10]$, and the fluid motion is described by various laws of flow in a porous medium, including the discrete element method or combination of DEM and FEM [11, 12]. Such formulations allow analyzing both the stress-strain behavior and fluid distribution in rock mass, as well as the fluid flow rates, leeks, etc.

Diverse packer designs commonly represent a body with an installed packer for airtight packing of boreholes. The packer-borehole wall interaction analysis is a relevant problem also sufficiently addressed. The scope of the studies in [13-15] embraces the finite element method-based numerical analysis of interaction between a rubber packer, packer components and a casing pipe, relationship between the application stresses and maximum shear stresses in loading of rubber hoses by fixed longitudinal displacement of expandable coupling, as well as the friction coefficients of rubber and the packer components.

\subsection{Boundary value problem formulation}

This paper presents the numerical results on loads applied to the borehole wall by the packers when expanding under the action of internal pressure, with regard to friction between the borehole wall and the packers. The influence of the length and spacing of the packers on the stress state in rock mass surrounding the borehole was also analyzed. The three-dimensional axially symmetric problem was solved using the finite element method within the classical theory of elasticity in COMSOL Multiphysics ${ }^{\circledR}$ envrionment [16]. The rock and packers are different modulus elastic materials. Loading is exerted by the pressure applied to the inside surface of the packers. In interaction between the packers and borehole wall, the contact is introduced in terms of the external dry friction angle $\varphi=30^{\circ}$. Figure 1 depicts the section of the test domain by the plane $\operatorname{Orz}$, where $O z$ is the symmetry axis in the space $\operatorname{Or} \theta z$.

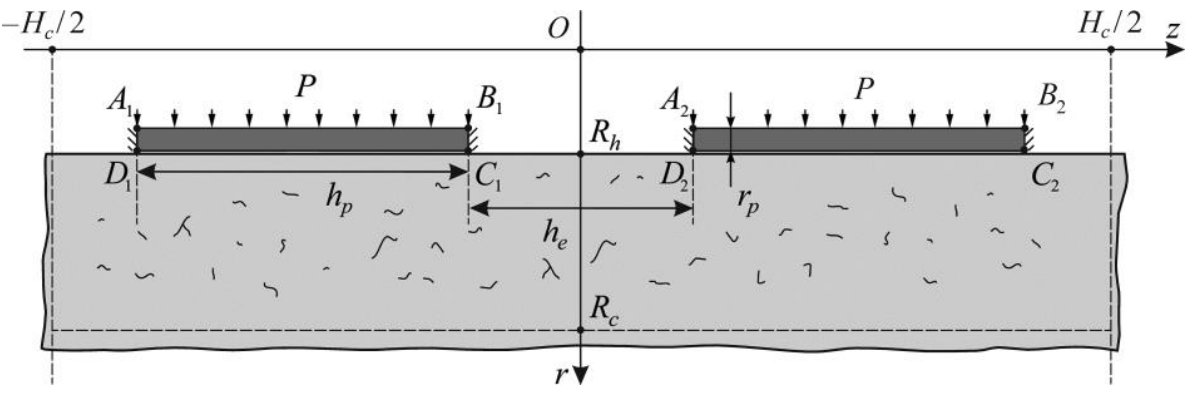

Fig. 1. Pattern of numerical calculation and conditions of loading. 
The rock mass domain has the dimensions $H_{c}=2.0 \mathrm{~m}$ and $R_{c}=1.0 \mathrm{~m}$ along the axes $\mathrm{Oz}$ and $O r$, respectively; the borehole radius is $R_{h}=0.05 \mathrm{~m}$. Two packers with the length $h_{p}$ are arranged symetrically relative to the axis $O r$ at the spacing $h_{e}$ in-between. The rubber of the packers has the thickness $r_{p}=15 \mathrm{~mm}$. There is no any gap between the packers and the borehole wall.

The physical parameters of the test materials are as follows. Rock is an elastic material with the Young modulus $E_{c}=5.4 \mathrm{GPa}$ and Poisson's ratio $v_{c}=0.16$, characteristic of coal. Packers are reinforced rubber with the Young modulus $E_{p}=50 \mathrm{MPa}$ and Poisson's ratio $v_{p}=0.45$.

Regarding the loading conditions, the internal boundaries $A_{1} B_{1}$ and $A_{2} B_{2}$ are applied with the preset and gradually increasing pressure $P$ from 0 to $10 \mathrm{MPa}$. The borehole wall $r=\underline{R}_{h}$ beyond the contact with the packers is free from stresses. At the ends of the packers (surfaces $A_{1} D_{1}, B_{1} C_{1}, A_{2} D_{2}, B_{2} C_{2}$ ), the condition of rigid fixing is set: $\mathbf{u}(r, z) \equiv 0$, where $\mathbf{u}(r, z)$ is the displacement vector. Some subcurves enveloping rock are also subjected to the condition of rigid fixing:

$$
\begin{gathered}
\mathbf{u}(r, z) \equiv 0 \text { when } r=R_{c} \text { and }-0.5 H_{c} \leq z \leq 0.5 H_{c}, \\
\mathbf{u}(r, z) \equiv 0 \text { when } R_{h} \leq r \leq R_{c} \text { and } z= \pm 0.5 H_{c} .
\end{gathered}
$$

Under loading by the internal incremental pressure $P$, the packers expand and their contact with the borehole wall grows. The computation series included different lengths and varied spacing of the packers: $h_{p}=40,60,80$ and $100 \mathrm{~cm} ; h_{e}=10,20,30$ and $40 \mathrm{~cm}$.

\subsection{Numerical results}

Rocks possess different strengths in compression and tension. The tension strength can be less than the compression strength by an order of magnitude. Our interest lies in the geomaterial areas dominated by the tensile stresses.

Let us consider the stress-strain behavior of the medium in the vicinity of the borehole at the final stage of loading. The calculations show that the tensile stresses are the axial stresses $\sigma_{z}$ generated in the domain which is limited along the axis $O z$ by the packers ($\left.h_{e} / 2<z<h_{e} / 2\right)$ and adjoins the borehole wall. Figure 2 illustrates the pattern of the axial stresses at the packer spacing $h_{e}=20 \mathrm{~cm}$. It is seen that even with no fracturing fluid fed in the free space between the packers, rock experiences extension under the action of forces generated by the packers.

a

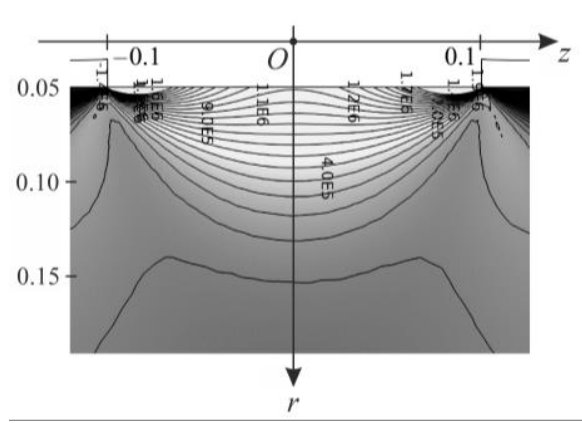

b

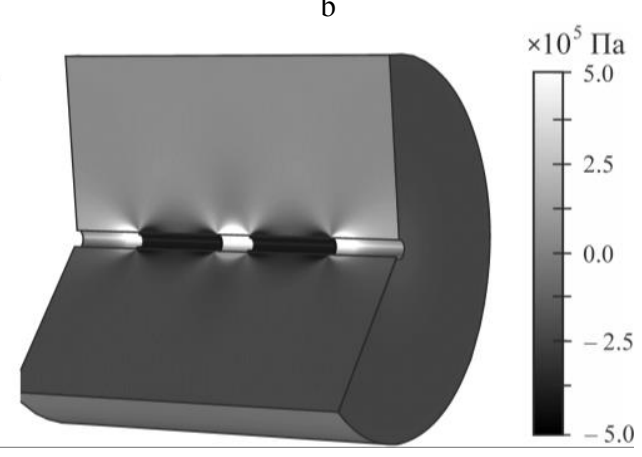

Fig. 2. Pattern of axial stresses $\sigma_{z}$ at the final stage of loading: (a) section by plane Orz; (b) 3D loading pattern. 
The behavior of the stresses at a distance from the borehole wall is analyzed using the stress diagram in the section $z=0$ at $R_{h} \leq r \leq R_{c}$. Figure 3 demonstrates the diagrams of the radial stress $\sigma_{r}$ and axial stress $\sigma_{z}$ at different packer-to-packer spacing $h_{e}$. The peak values of the radial tensile stress $\sigma_{r}$ is observed nearby the borehole wall (at a distance from 8 to $15 \mathrm{~cm}$ ), and the stress gradually lowers from $0.53 \mathrm{MPa}$ at $h_{e}=10 \mathrm{~cm}$ to $0.11 \mathrm{MPa}$ at $h_{e}=40 \mathrm{~cm}$ (Fig. 3a). The axial stress $\sigma_{z}$ reaches the maximum values at the borehole wall and reduces from 0.42 to $2.6 \mathrm{MPa}$ at $h_{e}=40 \mathrm{~cm}$ and $h_{e}=10 \mathrm{~cm}$, respectively (Fig. $3 \mathrm{~b}$ ).

a

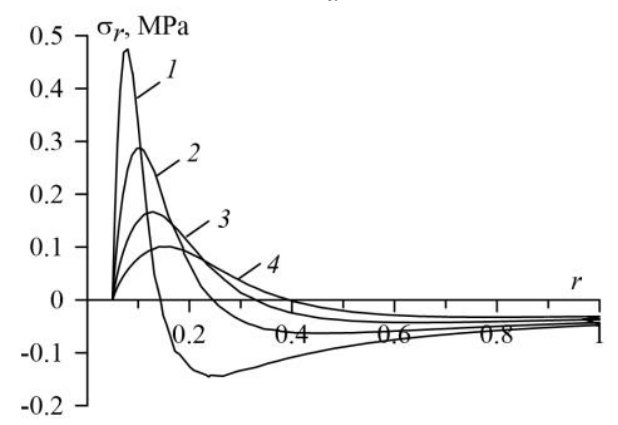

$\mathrm{b}$

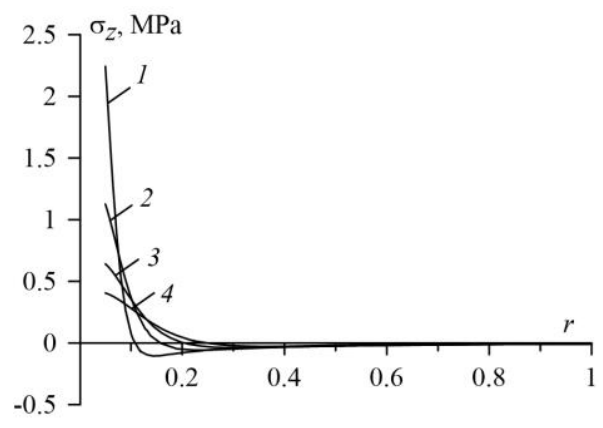

Fig. 3. Diagrams of (a) radial $\sigma_{r}$ and (b) axial $\sigma_{z}$ stresses in cross-section $z=0$ at packer-to-packer distances of $10(1), 20(2), 30(3)$ and $40 \mathrm{~cm} \mathrm{(4).}$

The influence of the packer-to-packer spacing on the stresses at the borehole wall is illustrated in Fig. 4. The values of the maximum axial tension $\sigma_{z}$ of the borehole wall are presented at the packer lengths $h_{p}$ of 40,60,80 and $100 \mathrm{~cm}$ (plotted on the abscissa) and at the different packer spacing $h_{e}=10,20,30$ and $40 \mathrm{~cm}$ (curves 1,2,3 and 4, respectively). It appears that as the spacing between the packers grows, the maximum tensile stresses at the check point decrease while the length of the packers has no essential effect on the values of $\sigma_{z}$.

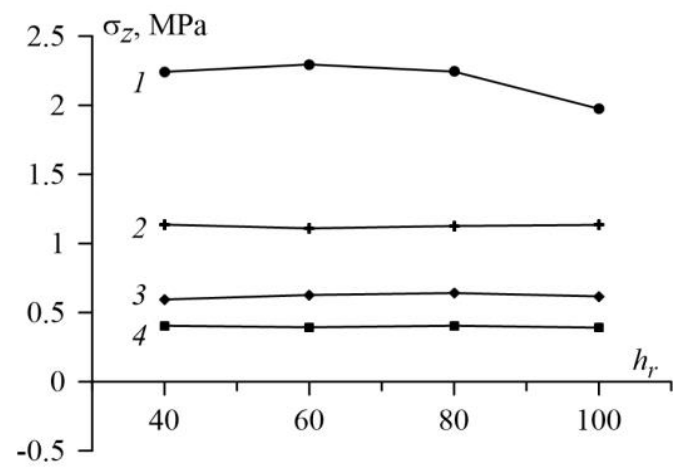

Fig. 4. Maximum tensile stress $\sigma_{z}$ on borehole wall at different packer lengths $h_{r}$ (abscissa) and packer-to-packer spacing $h_{e}=10(1) ; 20$ (2); 30 (3) and $40 \mathrm{~cm}$ (4).

Thus, the presented pattern of calculation by loading steps with regard to the contact between the reinforced elastic packers and borehole wall makes it possible to describe the pressure distribution at the rock-packer interface and to analyze the stress-strain behavior of rock mass at the preset working pressure in the packers versus different lengths and spacing of the packers. 


\section{Conclusions}

The authors have addressed the issue of in-situ methane drainage from coal seams using multiple hydraulic fracturing. The finite element method-based computational model presented in this paper allows analyzing the contact interaction between the borehole wall and the packers during expansion of the packers under the action of the internal pressure, with regard to the packer-rock friction, as well as enables assessment of the stress-strain behavior of rock mass in the vicinity of the borehole. It is shown that loading generates pressure on the contact surface, and this pressure induces essential axial tensile stresses in rocks in the vicinity of the borehole. This fact should be included in the boundary problem formulations.

The obtained results are applicable in selecting in-situ hydraulic fracturing design patterns and equipment to stimulate methane drainage from coal seams using uncased drainage boreholes drilled from underground openings.

The study was supported by the Russian Science Foundation, project no. 17-17-01143.

\section{References}

1. F. Jun, D. Linming, et al., J. Min. Sci. and Tech., 22(2), 177-181 (2015)

2. J. Deng, Q. Yang, et al., Computers and Geotechnics, 94, 118-133 (2018)

3. R. Jeffrey, K.Mills, X. Zhang, Proc. $3^{\text {rd }}$ Int. Workshop on Mine Hazards Prevention and Control (Brisbane, 2013)

4. V. Klishin, S. Klishin, A. Tatsienko, E3S Web of Conferences, 174 (2020)

5. S.V. Klishin, V.I. Klishin, Journal of Mining Science, 56(4), 547-556 (2020)

6. W. El Rabaa, SPE Annual Technical Conf. and Exhibition, Soc. of Petroleum Engineers (1989)

7. Z.J. Chen, Petroleum Sci. and Eng., 88, 136-144 (2012)

8. M. Wangen, Computational Geosciences, 17(4), 647-659 (2013)

9. Y. Zhao, S. Cao, et al., Computers and Geotechnics, 111, 229-242 (2019)

10. X. Zhang, R.G. Jeffrey, Thiercelin M. J. of Structural Geol., 29(3), 396-410 (2007)

11. Q. Zhao, A. Lisjak, et al., J. Rock Mech. and Geotech. Eng., 6(6), 574-581 (2014)

12. J.A.R. Cordero, E.C.M. Sanchez, et al., Comp. and Geotechnics, 111, 290-300 (2019)

13. Guo Z., Wang Y., et al., Advances in Mechanical and Electronic Engineering, (Springer, Berlin, Heidelberg, 2012)

14. W. Ma, B. Qu, F. Guan, Proc. of the Institution of Mech. Eng., Part C, J. Mech. Eng. Sci., 228(16), 2881-2887 (2014)

15. W.J. Lan, H.X. Wang, et al., 16(3), 632-644 (2019)

16. COMSOL Multiphysics ${ }^{\circledR}$ Modeling Software, https://www.comsol.com 